\title{
CIENCIA Y GOBERNANZA DE ECOSISTEMAS CON ÁREAS NATURALES PROTEGIDAS: EL EJEMPLO DE LOS EVERGLADES (EE.UU.)
}

\author{
Carol L. Mitchell \\ Investigadora independiente y voluntaria de la Oficina de Asuntos \\ Internacionales del Parque Nacional Everglades, Estados Unidos
}

\begin{abstract}
Resumen: La ciencia es clave para asumir el reto principal del manejo de ecosistemas para el desarrollo sostenible: ¿cómo lograr los beneficios que resultan de la transformación y explotación de ecosistemas, y al mismo tiempo conservar los servicios ecosistémicos y las áreas naturales protegidas? En el ecosistema de los Everglades, humedal subtropical localizado en el sur de Florida (Estados Unidos), ocurrió una transformación económica y ambiental a mediados del siglo XX. Hoy, el Programa de Restauración de los Everglades está abordando el reto de mantener los beneficios del ecosistema transformado y, al mismo tiempo, proteger y restaurar el Parque Nacional Everglades. Un análisis de la ciencia y gobernanza del Programa de Restauración de los Everglades nos permite entender este proceso. Las decisiones fuertes sobre el programa son tomadas por una comisión especial diversa, compuesta al nivel más alto del gobierno, equivalente al nivel de ministros. El rol de la ciencia en el diseño, ejecución y monitoreo está escrito dentro de la ley y reglamento que rige el Programa. Finalmente, todas las agencias miembros de la Comisión emplean técnicos y científicos calificados, cuyo trabajo es el de hacer, recopilar y sintetizar investigaciones científicas. Este sistema reduce la distancia entre los científicos y los tomadores de decisiones. Al mismo tiempo, el sistema de gobernanza del Programa permite que los actores con menor poder financiero o político puedan ejercer poder por medio de sus resultados científicos, logrando así un balance entre los beneficios económicos y los beneficios ambientales del Programa.
\end{abstract}

Palabras clave: Ciencia. Tomadores de decisiones. Everglades. Gobernanza. Áreas protegidas. 


\title{
Science and Governance of Ecosystems and Parks: the Everglades (USA) case study.
}

\begin{abstract}
Science is key to solving the challenge of managing ecosystems for sustainable development. How do we achieve the benefits that result from the transformation and exploitation of ecosystems, and at the same time preserve ecosystem services and protected areas? In the ecosystem of the Everglades, a subtropical wetland located in South Florida (United States), an economic and environmental transformation occurred in the middle of the 20th century. Today, the Everglades Restoration Program is addressing the challenge of maintaining the benefits of the transformed ecosystem while at the same time protecting and restoring Everglades National Park. An analysis of the governance of this program highlights three important aspects of the system with respect to science. First, the primary decision-making body is the South Florida Ecosystem Restoration Task Force, a diverse group of 14 agency leads at the highest level of national, tribal, state and local government. Second, the role of science in the restoration program is written into the federal laws and regulations governing the program. Finally, all the member agencies of the Task Force employ qualified technicians and scientists whose work is to conduct, collect and synthesize scientific research. This system reduces the distance between scientists and decision makers. At the same time, the program's governance system allows actors with less financial or political power to exercise power through their scientific results, thus achieving a balance between the economic benefits and environmental benefits of the program.
\end{abstract}

Keywords: Science. Decision-makers. Everglades. Governance. Protected areas.

\section{Carol L. Mitchell}

Recibió su Bachillerato en Zoología de la Universidad de California y su Ph.D. en Ecología y Biología de la Evolución de Princeton University. De 1983 a 2003 realizó trabajos de investigación y conservación en Madre de Dios, Perú. De 2004 a 2018 fue Sub-Directora de Ciencias en el Parque Nacional Everglades, con el Servicio de Parques Nacionales de los Estados Unidos (NPS, por sus siglas en inglés). Ha trabajado en varios países de América Latina y el Caribe, así como en el sur y sudeste de Asia. Actualmente es consultora independiente y voluntaria de la Oficina de Asuntos Internacionales del NPS.

Correo electrónico: Cmbluewing@bellsouth.net 


\section{Introducción}

La ciencia es una herramienta de suma importancia para abordar el reto del manejo de ecosistemas para el desarrollo sostenible. Este reto existe en todos los países del mundo. ¿Cómo lograr los beneficios que resultan de la transformación de ecosistemas y al mismo tiempo conservar los servicios ecosistémicos que benefician a la sociedad local y a la sociedad mayor? Específicamente, ¿cómo lograr el desarrollo económico deseado con la construcción de megaproyectos de infraestructura o energía, agricultura o extracción forestal, o de desarrollo urbano, y al mismo tiempo proteger servicios ecosistémicos como la regulación natural de aguas de cuencas, de recursos naturales de importancia como los recursos pesqueros, y lugares naturales que conservan la biodiversidad y proporcionan oportunidades para el uso tradicional, la educación, el recreo y el descanso para las poblaciones locales y la sociedad mayor? En los ecosistemas que albergan las áreas naturales o áreas culturales protegidas declaradas de importancia local y nacional este reto es aún más complicado. Todas las áreas protegidas se ubican dentro de los ecosistemas, y el manejo y el éxito de las áreas naturales protegidas depende mucho de su entorno ecosistémico.

En el Perú hay varios factores que están impulsando actualmente una transformación muy rápida y amplia de áreas naturales y de ecosistemas. Esta transformación se siente mucho en la Amazonia peruana, lugar donde se ubica la mayor extensión de áreas naturales protegidas por el Estado. Así tenemos que 9 de los 15 parques nacionales peruanos (más del 90\% de las hectáreas protegidas por parques nacionales), 5 de 15 reservas nacionales (más del 70\% de las hectáreas protegidas por reservas nacionales) y 7 de 10 reservas comunales (80\% de las hectáreas de reservas comunales) en el Perú se ubican en la Amazonia (SERNANP 2018).

En los últimos veinte años, la ejecución de grandes proyectos de transporte y energía como el gas de Camisea y la carretera interoceánica, combinados con un período de estabilidad política y una creciente demanda en el exterior de recursos naturales energéticos, minerales, madereros y otros, están fomentando un período de crecimiento económico histórico en el país (Andina 2017). Escogiendo una sola cifra económica perteneciente a la 
Amazonia peruana, el valor de las exportaciones de productos maderables se ha triplicado entre los años 2000-2015, de US\$68 millones a US\$215 millones (Ramírez Arroyo et al. 2012; Pacheco Roldán et al. 2016). Durante este mismo período, las tasas de deforestación en los bosques amazónicos del Perú han ido en crecimiento cada año (Global Forest Watch 2018; SERFOR 2015; Butler 2017). Aunque no es posible examinar en detalle aquí, es claro que las mejoras en la economía y la calidad de vida en el Perú están siendo acompañadas por la transformación física, ecológica, social y cultural de los ambientes naturales, en especial las áreas naturales de la Amazonia.

En el diseño de los megaproyectos es normal y requerido contar con el uso de información técnico/científica y con ingeniería de último momento; asimismo, con información cuantitativa, científica y técnica sobre las inversiones agrícolas, la extracción forestal y minera, y otras actividades que transforman el medio ambiente, para que estas puedan lograr sus objetivos económicos. Es menos conocido y menos esperado, pero no menos importante, el uso de la ciencia en la planificación, diseño y manejo de las áreas protegidas y en el manejo de ecosistemas con usos múltiples. Lo ideal es considerar ambos al mismo tiempo, juntar los aspectos técnico/científicos de los grandes proyectos de infraestructura y de producción, con lo que nos dice la ciencia sobre el entorno ambiental y en especial sobre las áreas naturales protegidas. Este proceso teóricamente debe suceder durante el Estudio de Impacto Ambiental (EIA); sin embargo, hay casos de proyectos cuyos EIA han resultado ser insuficientes para la protección del medio ambiente.

Entonces, ¿cómo se organiza un sistema de producción y diseminación de ciencia para el manejo de ecosistemas, poniendo importancia en la ciencia para la conservación y las áreas naturales protegidas? ¿Cómo se puede estar mejor preparados para proveer de la ciencia necesaria a las personas más importantes y en el momento oportuno?

Es claro que el diseño de un sistema exitoso de provisión de ciencia tiene que tomar en cuenta aspectos particulares de cada situación. ¿Cuál es el sistema de gobernanza en la toma de decisiones sobre el ecosistema, sobre los recursos naturales o sobre el área o áreas protegidas? ¿Cuáles son los cargos con poder real sobre las decisiones? ¿Los tomadores de decisiones son personas con experiencia en la ciencia, o son personas con otros estudios y otras historias? ¿Quiénes están produciendo la ciencia pertinente?

Las siguientes páginas exploran dos creencias comunes con respecto a la ciencia y a la toma de decisiones, y proporcionan información que da textura y detalle a la relación entre la ciencia y los tomadores de decisiones. Luego se describe la situación del sur de la Florida en los Estados Unidos y el Programa de restauración del ecosistema de los Everglades, donde la ciencia es fundamental y es demandada y utilizada por los principales responsables 
de la toma de decisiones, en beneficio de la sociedad y el medio ambiente. Para ello se han extraído algunas características del ejemplo de los Everglades que son determinantes en términos de su éxito en lograr resultados equilibrados; es decir, en proporcionar beneficios de un entorno transformado para la población local, al mismo tiempo que mantiene y restaura el sistema natural.

\section{1. ¿Ciencia para quiénes? El mundo de los tomadores de decisiones}

Desde hace años, los científicos han lamentado la dificultad de hacer llegar su información a los tomadores de decisiones (Parlamient U.K. 2012) e incluso han descrito esfuerzos activos para evitar o eliminar el uso de la ciencia en las decisiones de políticas ambientales (Carter et al. 2018). Estas críticas reflejan la idea, muy concretada en el mundo de los científicos, de que la ciencia es tan importante que los tomadores de decisiones deberían estar activamente buscándola y utilizándola en su trabajo. En los últimos veinte años, en diferentes países y organizaciones internacionales se han desarrollado varias estrategias intentando resolver esta situación (Fischhoff 2011; Piotrowski 2014). Los académicos han inventado sistemas de comunicación de la ciencia, que incluyen estándares de iconos y dibujos orientados a demostrar algunos de los procesos complejos de ecología y ecosistemas (Lookingbill et al. 2007; University of Maryland Center for Environmental Science 2018). Se han desarrollado modelos, artículos, libros, cursos y hasta carreras académicas enteras, sobre el tema de comunicación de la ciencia hacia los tomadores de decisiones (Jacobson 1999; von Winterfeldt 2012; Stevens Institute of Technology s.a.; Universidad Autónoma de Madrid 2017).

En este esfuerzo, los científicos se concentran en lo que conocen: la ciencia y los asuntos académicos. Pero, ¿cómo hacemos para que la información científica tenga impacto? Para abordar esta pregunta, hay que analizar el mundo de los tomadores de decisiones, enfocándonos para fines de este artículo en los que trabajan en temas de uso o transformación de los recursos naturales, en el manejo de ecosistemas y en áreas naturales protegidas (Ver Figura 1). La persona que está al centro de la figura puede ser cualquier persona que esté encargada de decisiones que afectan los ecosistemas y los recursos naturales: jefe de parque, congresista, líder indígena, ministro/a, u otro. Muchas veces, esta persona no tiene estudios científicos: puede ser político, administrador, economista, empresario/a, o cualquier otra carrera que no sea científica; puede no tener ningún estudio formal, pero es una persona de importancia para su comunidad y para la gestión de los recursos naturales. Generalmente son personas expertas y 
exitosas dentro del tema de su conocimiento, de cual tema los científicos generalmente son ignorantes.

En su capacidad de tomadores de decisiones, estas personas a diario tienen que lidiar con una serie de factores que no necesariamente incluyen la ciencia. Muchas veces están encargados de grupos de empleados o personas, están encargados de presupuestos, y tienen que prestar atención a los deseos de sus jefes y demás individuos de mayor rango. Para mantener su posición de líder tienen que tomar innumerables decisiones diarias de índole social y política. Es de mucha importancia que los científicos reconozcan estos factores en la vida de los tomadores de decisiones: la ciencia está compitiendo con un sinnúmero de otros temas en consideración. Como dijo el doctor Rush Holt, líder de la Asociación Americana del Avance de la Ciencia, y lo dijo con respeto: «Los formuladores de políticas no piensan en la ciencia con frecuencia, ni anhelan el asesoramiento científico. Ni siquiera saben qué preguntar» («Policy makers do not think of science frequently or crave scientific advice. They do not even know what to ask») (King 2016:1512).

Lo que es cierto es que la ciencia no necesariamente está en la vida diaria de los tomadores de decisiones. Aun cuando existe un marco formal para la entrega de la ciencia a los decisores (por ejemplo, por medio de una EIA), la ciencia entra solamente a veces y a veces entra en un momento inoportuno. Muchas veces cuando entra en consideración, la ciencia forma una parte muy pequeña de las decisiones. El reto para los científicos es el de proveer la información pertinente, en el momento más oportuno, conociendo los otros factores que también están involucrados en la decisión.

Figura 1.

\section{El tomador de decisiones rodeado de las preocupaciones diarias}

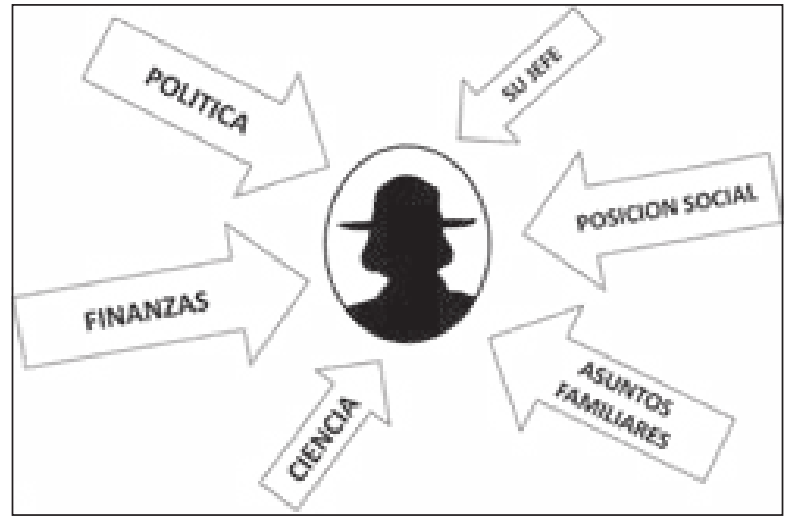




\section{El mundo de la producción y entrega de la ciencia}

Es muy común concebir a la ciencia como una actividad restringida a las universidades, a los pocos institutos del gobierno dedicados a esta actividad, o a entidades privadas como laboratorios analíticos o de investigación con fines de lucro. Lo más simple, pero tal vez el concepto más común, es concebir al proceso de entrega de información a los tomadores de decisiones como un proceso lineal: se produce la ciencia en las universidades, y esa ciencia hay que llevarla y traducirla para los tomadores de decisiones.

Este concepto teórico de proceso lineal conlleva a las propuestas de desarrollo de herramientas de decisión. Las herramientas de decisión en su forma más simple generalmente son modelos cuantitativos simplificados en computadora, con una interfaz gráfica del usuario y que son diseñados específicamente para el uso de los tomadores de decisiones. En estas propuestas, el desarrollo de la herramienta de decisión generalmente va acompañado por propuestas de talleres de capacitación, donde los tomadores de decisiones serían entrenados en el uso y aplicación de tales herramientas en las decisiones que pudieran tomar. ${ }^{1}$

En realidad, los tomadores de decisiones pueden recibir información científica (e información no científica) de varias fuentes, no solamente de los académicos o quienes han producido los resultados originales. Otras agencias del gobierno, las organizaciones no gubernamentales, las organizaciones financieras, el público y otros actores tienen acceso al tomador de decisiones. Todos estos actores son capaces de recoger resultados científicos, de resumirlos y de comunicarlos a los tomadores de decisiones por varios medios. Esta realidad es compleja. El marco de participación de estos actores - sea formalmente establecido o informalmente ejecutado- afecta mucho la probabilidad de que el tomador de decisiones reciba la información adecuada en el momento adecuado.

Vale decir que, no obstante el origen de la investigación científica o el camino que recorren los resultados para llegar al tomador de decisiones, el líder y el público necesitan tener confianza en que los resultados sean imparciales. Al mismo tiempo, el tomador de decisiones tiene que confiar en el mensajero. La ciencia más importante, más oportuna y mejor ejecutada puede no tener impacto si la fuente de la información no es de confianza del tomador de decisiones.

1 Para ejemplos de este concepto, ver IDEAM et al. 2015 y USAID-SERVIR 2017. 


\section{La ciencia y la gobernanza en la restauración de los Everglades en el Sur de la Florida, Estados Unidos}

En el sur del estado de la Florida, en los Estados Unidos, existe el ecosistema de los Everglades, un humedal de más de $46.000 \mathrm{~km}^{2}$. La cuenca hidrológica del ecosistema cubre casi la tercera parte del estado de la Florida, un paisaje casi del tamaño del departamento de San Martin o la mitad del departamento de Madre de Dios, en el Perú. En este ecosistema se reúnen características naturales, culturales, sociales y políticas que lo hacen apto para una demostración del papel de la ciencia y la gobernanza en el manejo de ecosistemas con áreas naturales protegidas.

El ecosistema de los Everglades alberga una variedad de hábitats, desde bosques de pino, humedales de agua fresca, los manglares más extensos del hemisferio oeste hasta zonas marinas ricas en diversidad y en recursos pesqueros (Ver Figura 2). La importancia de esta área para la conservación de la naturaleza en los Estados Unidos es tal que existen cuatro parques nacionales, un santuario marino nacional, reservas estatales de aguas, reservas indígenas y varios parques estatales y municipales, cubriendo casi la tercera parte del sur de la península. Contando solo las áreas protegidas a nivel nacional, casi una de cada cinco hectáreas del sur de Florida recibe este nivel alto de protección. De estos parques, el Parque Nacional Everglades es el más grande ( $>600,000$ has), más conocido y más amado por el público estadounidense.

Al mismo tiempo, este ecosistema alberga más de seis millones de personas, incluyendo el área metropolitana de Miami, Fort Lauderdale y West Palm Beach, donde ha existido un crecimiento poblacional de casi un $20 \%$ por década durante los últimos ochenta años. Asimismo, existen áreas agrícolas de importancia, que producen caña de azúcary verduras para el país y para la exportación. Es lugar de importancia para la industria de cruceros, la industria naviera y de puertos marítimos. El turismo y las actividades de recreación en la naturaleza contribuyen fuertemente a la economía local. La pesca deportiva, como actividad única, trae un impacto económico de más de $\$ 1.000$ millones USD anuales (Fedler 2009). 
Figura 2.

Mapa del sur de Florida, mostrando las principales áreas urbanas y áreas naturales protegidas

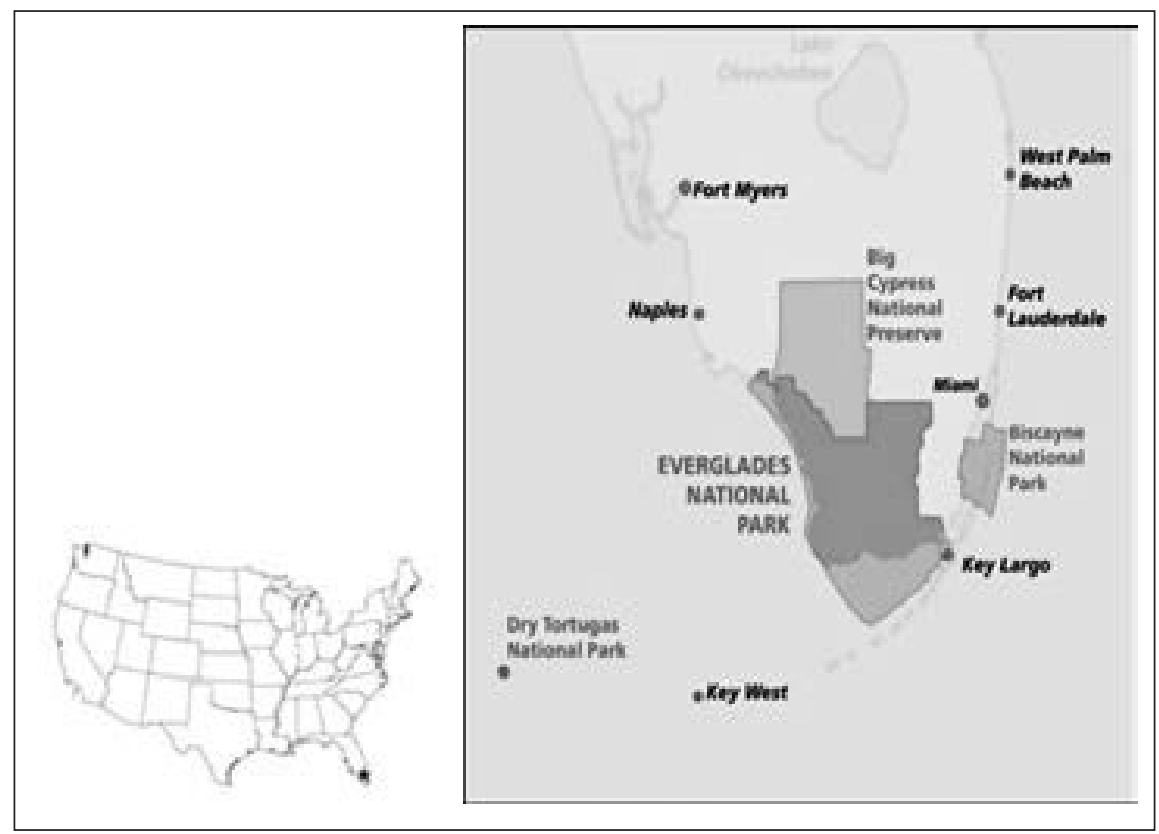

Fuente: South Florida Natural Resources Center, Everglades National Park. Elaboración propia.

La situación actual del sur de la Florida está ligada fuertemente a la construcción de un vasto sistema de manejo de aguas superficiales, proyecto que fue realizado durante casi ochenta años, desde 1890 hasta 1970. Es un sistema de más de mil kilómetros de canales y diques, de cientos de compuertas y bombas de agua que remueven más de dos mil millones de galones de agua fresca de la superficie del sur de la Florida a diario. Es un megaproyecto inmenso, cuya instalación demoró casi un siglo y sobre cuyo funcionamiento eficiente yace la economía de esta área. Este sistema de manejo de aguas ha transformado el humedal natural en áreas agrícolas, industriales, urbanas y residenciales, protegiendo estas zonas de uso humano contra las inundaciones periódicas que son naturales en los ecosistemas de humedales. El sistema provee agua para la agricultura y agua potable para la población, y hace posible la existencia del área metropolitana de Miami, Fort Lauderdale y West Palm Beach (SFWMD s.a.). 
La culminación de la construcción de este sistema de manejo de aguas superficiales transformó el paisaje natural de los Everglades a mitades del siglo XX, trayendo consigo consecuencias negativas para los recursos naturales. El área del Parque Nacional Everglades recibe menos de la mitad del agua superficial que recibía esta área antes del megaproyecto. Las aguas superficiales de los Everglades hoy en día no muestran las características históricas de creciente y vaciante, tan importantes para la flora y fauna nativa del área. Se estima que las poblaciones deaves zancudas (garzas, ibis y cigüeñas, elementos claves del ecosistema) han disminuido a solo el $10 \%$ de su nivel histórico, antes del proyecto. Las acequias artificiales creadas por el sistema de aguas están relacionadas con una alta frecuencia de incendios de pastizales y bosques. El ecosistema de humedal, naturalmente bajo en nutrientes, sufre por la carga de fertilizantes agrícolas, y demás entradas de nitrógeno y fósforo a las aguas superficiales y costeras, resultando en floraciones de algas nocivas que abaten a la flora y fauna nativa, y afectan hasta la salud de las personas quienes residen cerca (Grunwald 2006; The Everglades Foundation 2018).

A raíz de estos impactos, en el año 2000 se aprobó el Programa para la Restauración de los Everglades, un esfuerzo financiado 50/50 entre el gobierno nacional y estatal, y apoyado en su forma conceptual por la mayoría de actores del área. El propósito de este programa es complejo: restaurar características hidrológicas históricas del ecosistema, restaurar la fauna y flora de los parques y reservas, restaurar los servicios ecosistémicos para la sociedad (el agua limpia, la pesca abundante, las oportunidades para recreo y descanso, y con eso, el turismo), y al mismo tiempo mantener las protecciones contra las inundaciones, y mantener la provisión de agua para la agricultura y para la gente que vive en la zona. Esta dualidad de restauración del ecosistema natural junto con la protección de aspectos del ecosistema transformado, es el gran reto para la ciencia y para la sociedad.

Todos los megaproyectos están basados en la ingeniería y la ciencia desde un principio, enfocados en lograr el objetivo del megaproyecto, sea transporte terrestre o fluvial, o energía eléctrica, extracción de petróleo, protección de las inundaciones, u otro objetivo social y financiero. Tanto el megaproyecto de manejo de aguas superficiales de los Everglades, como el megaproyecto de restauración, tienen bases en la ingeniería hidrológica. En un ecosistema de humedal, la hidrología es la base de toda la red de vida, conectando de forma física, química y biológica el agua, las algas y las plantas acuáticas, los invertebrados y peces pequeños, los peces mayores, las aves zancudas, los lagartos y demás habitantes silvestres del ecosistema. Este hecho abre caminos para la participación no solamente de la ingeniería hidrológica, sino también de las ciencias químicas, biológicas y ecológicas en el Programa de Restauración. 
En el Programa de Restauración de los Everglades, hay tres temas cuyo tratamiento aumenta las posibilidades de éxito, es decir, el lograr los fines duales del Programa. Como una base, es obligatorio seguir la Ley NEPA ${ }^{2}$ de 1970, ley que rige los Estudios de Impacto Ambiental (EIA) en los EE.UU. en el desarrollo de todos los proyectos y actividades comprendidas en el Programa. NEPA tiene un papel similar a la Ley peruana $\mathrm{N}^{\circ} 27446$ de 2013, que creó el Sistema Nacional de Evaluación del Impacto Ambiental (SEIA). Los temas expuestos a continuación son adicionales a los requisitos de análisis NEPA (los EIA), y proveen un marco más complejo, pero más completo para la incorporación de información científica en el Programa de Restauración de los Everglades.

Gobernanza participativa y de alto nivel: el Programa de Restauración de los Everglades fue aprobado por la leyes «Water Resources Development Act 1996 y 2000» (The 106th Congress, 2000). Está supervisado por la Comisión Especial de la Restauración del Ecosistema del Sur de Florida. Esta Comisión tiene catorce miembros, de cuatro entidades gubernamentales (Ver Cuadro 1). La Ley de 1996 determina que la participación oficial en la Comisión es al nivel de las secretarías de los departamentos del gobierno federal (equivalente al nivel de ministro), al nivel más alto estatal (Gobernador de la Florida), al nivel más alto de las naciones indígenas locales (Jefe de Tribu), y al nivel de los otros gobiernos locales del sur de Florida. La Comisión se encarga de evaluar el Programa dos veces al año. Un papel de mucha importancia de esta Comisión es la resolución de conflictos en el diseño y la ejecución de los varios y costosos proyectos incluidos en el programa (SFERTF s.a.).

La ciencia es reconocida en la Ley y Reglamento del Programa de Restauración: la Ley y el Reglamento del Programa establecen no menos de tres entidades con el papel formal de informar a la Comisión sobre resultados científicos pertinentes. El primer grupo se llama RECOVER, conformado por técnicos y científicos empleados en las catorce entidades miembros de la Comisión. RECOVER es el grupo netamente técnico, encargado tanto de proveer de herramientas aptas para el diseño de proyectos, como de monitorear los aspectos claves del ecosistema y reportar sobre avances y problemas encontrados. Con este fin, las diferentes agencias manejan no solamente empleados propios, sino también fondos para la ejecución de contratos y acuerdos de investigación con universidades y otras entidades técnicas. RECOVER está vinculado cercanamente con los EIA para cada proyecto dentro del Programa; la información científica producida por RECOVER es incorporada en los EIA. Sin embargo, el papel más importante de RECOVER es de analizar al nivel de Programa, de evaluar científicamente

2 National Environmental Policy Act, 1970. 
si la suma de los diferentes proyectos y actividades está resultando en la restauración del ecosistema mayor.

\section{Cuadro 1.}

\section{Actores participantes en la Comisión Especial de la Restauración del Sur de Florida}

\begin{tabular}{|c|c|c|}
\hline Actor & $\begin{array}{l}\text { Número de } \\
\text { representantes }\end{array}$ & Nivel gubernamental \\
\hline Department of the Interior & 1 & Federal- Presidencia \\
\hline Department of Commerce & 1 & Federal \\
\hline $\begin{array}{c}\text { Department of the Army (Corps of } \\
\text { Engineers) }\end{array}$ & 1 & Federal \\
\hline Environmental Protection Agency & 1 & Federal \\
\hline Attorney General & 1 & Federal \\
\hline Department of Agriculture & 1 & Federal \\
\hline Department of Transportation & 1 & Federal \\
\hline $\begin{array}{c}\text { Miccosukee Tribe of Indians of } \\
\text { Florida }\end{array}$ & 1 & $\begin{array}{c}\text { Gobierno Indígena } \\
\text { (Native American } \\
\text { Tribe) }\end{array}$ \\
\hline Seminole Tribe of Florida & 1 & $\begin{array}{c}\text { Gobierno Indígena } \\
\text { (Native American } \\
\text { Tribe) }\end{array}$ \\
\hline State of Florida (Governor's office) & 2 & Estatal (State) \\
\hline $\begin{array}{c}\text { South Florida Water Management } \\
\text { District }\end{array}$ & 1 & Estatal (State) \\
\hline $\begin{array}{l}\text { Local Governments of South } \\
\text { Florida }\end{array}$ & 2 & Provincial (County) \\
\hline
\end{tabular}

Fuente: www.evergladesrestoration.gov. Elaboración propia

El segundo grupo es el Grupo de Coordinación Científico, conformado por representantes de nivel alto de las catorce entidades. Este Grupo se encarga de identificar temas científicos que requieren atención, se encarga de resolver disputas técnicas entre los científicos de las diferentes agencias y otras entidades de la Comisión, y está encargado del aspecto técnico/ científico de los reportes de la Comisión al Congreso de los Estados Unidos. 
Finalmente, la Ley requiere la participación de la Academia Nacional de Ciencias de los Estados Unidos, el grupo más alto de asesoría científica para el gobierno federal. La Academia tiene un papel de evaluación independiente a grandes rasgos, con por lo menos dos reuniones públicas al año y un reporte cada dos años. En contraste con RECOVER, los miembros de la Academia no son empleados de las agencias miembros de la Comisión; tampoco reciben fondos de investigación por parte del Programa. Su papel es de evaluación independiente a nivel macro.

En cada agencia hay científicos cerca de los tomadores de decisiones: existen puestos técnicos y científicos dentro de cada agencia miembro de la Comisión. Algunas agencias, en especial las que ejecutan los proyectos, tienen grupos de decenas de científicos de varias especialidades para proveer la información técnica requerida. Algunas instituciones tienen solo un puesto, pero ese puesto es dedicado a entender la ciencia pertinente e informar a sus superiores. El Servicio de Parques Nacionales, dentro del Parque Nacional Everglades, tiene un grupo de veinte a veinticinco puestos técnicos dedicados al Programa de Restauración. El papel de estos científicos es el de participar en los aspectos técnicos del Programa, producir información técnica que ayuda al Servicio de Parques a lograr su misión de conservación, e informar a los tomadores de decisiones de su agencia y departamento. La ubicación de los científicos dentro de las agencias tiene la ventaja de una cercanía al tomador de decisiones, lo que aumenta la probabilidad de que la información pertinente llegue al tomador de decisiones en el momento oportuno. Por parte de los científicos de agencia, hay mayor conocimiento del mundo del tomador de decisiones. ${ }^{3}$

\section{Conclusiones}

En el sistema de gobernanza del Programa de Restauración de los Everglades, la ciencia resulta ser una gran niveladora de poderes. Las agencias y tribus miembros de la Comisión varían tremendamente en cuanto a su presupuesto, el número de empleados dedicados al Programa, sus responsabilidades (algunos son ejecutores, otras son participantes), y su poder político. El papel central de la ciencia en este Programa y el sistema de gobernanza que crea espacios formales de participación de las agencias miembros de la Comisión en el debate técnico/científico, hace que los actores menos poderosos puedan lograr impactos positivos para sus misiones y el ambiente. Por ejemplo, el Cuerpo de Ingenieros del Ejército de los Estados Unidos es ejecutor del Programa, y

3 Recordar la Figura 1. 
su presupuesto anual para el Programa varía entre US\$60 millones a US\$120 millones. La misión del Cuerpo de Ingenieros es la de protección contra inundaciones y de provisión de aguas; es netamente económica y de mitigación de riesgos, y no es ecológica. En contraste, el Servicio de Parques Nacionales no es ejecutor, es participante en el Programa por medio de su agencia madre, el Departamento del Interior. El presupuesto del NPS para la restauración de los Everglades es de alrededor de US\$9 millones a US\$10 millones al año. Sin embargo, los resultados científicos producidos y diseminados por el NPS han afectado de manera positiva los resultados hidrológicos y ecológicos de los proyectos diseñados y ejecutados dentro del marco del Programa de Restauración de los Everglades, en bien de la protección del medio ambiente y los recursos naturales y culturales del Parque Everglades.

La ciencia continuará siendo un elemento de suma importancia mientras el Perú y otros países naveguen el camino de transformación y conservación de sus paisajes y recursos en busca del desarrollo sostenible. El sistema de gobernanza de los ecosistemas en transformación, especialmente los que albergan áreas naturales protegidas, es clave para lograr múltiples objetivos sociales, económicos y ecológicos de la actividad humana. En la gobernanza del Programa de Restauración de los Everglades resaltan tres características importantes que ayudan a mejorar el uso de la ciencia en decisiones sobre el ecosistema mayor. El nivel de los tomadores de decisiones es de lo más alto, así se asegura que las decisiones hechas tengan peso y sean acatadas. El papel de la ciencia en el sistema de gobernanza está escrito en la Ley del Programa y su Reglamento, dando estructura formal para la entrega y discusión de resultados científicos de los diferentes actores, no obstante su presupuesto o su poder político. Los puestos técnicos y científicos relacionados con el Programa de Restauración se ubican dentro de las agencias, acortando la distancia entre el científico y el tomador de decisiones.

La gobernanza del Programa de Restauración de los Everglades, y el papel central de la ciencia en el diseño y ejecución del Programa son un ejemplo, de un Programa grande, en un momento de la historia, y en un solo país. Dentro del marco mayor de los valores locales y nacionales de la sociedad, y de las leyes de protección y de desarrollo en el sur de la Florida, este sistema de ciencia y gobernanza funciona bien. Para lograr superar el reto de desarrollo sostenible a un nivel mayor y en más lugares, hay que prestar atención a los sistemas de ciencia y gobernanza que rigen el desarrollo y ejecución de los megaproyectos en ecosistemas con áreas naturales protegidas. Hay que diseñar con cuidado los espacios de articulación de intereses, y buscar oportunidades para la ciencia de ecosistemas — no solamente la ingeniería-en decisiones sobre el diseño, ejecución y operación de los megaproyectos. 


\section{Referencias}

ANDina (2017). «BCR: Perú cumplirá 20 años de crecimiento económico continuo». Agencia Peruana de Noticias, 23 de octubre. Recuperado de: https://andina. pe/agencia/noticia-bcr-peru-cumplira-20-anos-crecimiento-economicocontinuo-729528.aspx.

Butler, R. (2017). Calculating Deforestation in the Amazon. Recuperado de: https:// rainforests.mongabay.com/amazon/deforestation_calculations.html.

Carter, J., A. Markham, J. Clement, M. Heid y P. Worth (2018). Science under Siege at the Department of the Interior. Cambridge, MA. Recuperado de: http://www.ucsusa.org.

FEDLER, T. (2009). The Economic Impact of Recreational Fishing in the Everglades Region. Recuperado de: https://www.bonefishtarpontrust.org/downloads/ research-reports/stories/everglades-economics-report.pdf.

FischHofF, B. (2011). "Applying the science of communication to the communication of science». Climatic Change. Recuperado de: https://doi. org/10.1007/s10584-011-0183-9.

Global Forest Watch (2018). Peru: Country Dashboard. Recuperado de: https:// www.globalforestwatch.org/dashboards/country/PER.

Grunwald, M. (2006). The swamp: the Everglades, Florida, and the politics of paradise. New York: Simon \& Schuster.

IDEAM, PNUD, MADS, DNP, CANCILLERÍA (2015). Nuevos Escenarios de Cambio Climatico para Colombia 2011-2100: Herramientas Científicas para la Toma de Decisiones - Enfoque Nacional - Departamental: Tercera Comunicación Nacional de Cambio Climático. Bogotá. Recuperado de: http://modelos.ideam.gov.co/ media/dynamic/escenarios/documento-nacional-departamental-2015.pdf.

Jacobson, S. K. (1999). Communication Skills for Conservation Professionals. Washington, D.C.: Island Press.

KING, A. (2016). «Science, politics and policymaking: Even though expert knowledge has become indispensible for policymaking, providing scientific advice to governments is not always easy». EMBO Reports, 17(11), 1510-1512 pp. Recuperado de: https://doi.org/10.15252/embr.201643381.

Lookingbill, T., W. C. Dennison, T. J. B. Carruthers, J. M. Hawkey y S. L. Carter (2007). «An Eye-Opening Approach to Developing and Communicating Integrated Environmental Assessments». Frontiers in Ecology and the Environment, 5(6), 307-321 pp. Recuperado de. https://esajournals. onlinelibrary.wiley.com/doi/10.1890/1540-9295\%282007\%295\%5B3 07\%3AAEATDA\%5D2.0.CO\%3B2. 
Pacheco Roldán, D., C. Vásquez Vizarreta, e Y. Gallardo Véliz (2016). Anuario Forestal 2015. Lima. Recuperado de: http://www.serfor.gob.pe.

PARLAMiEnt U.K., House of LoRds (2012). The role and functions of departmental Chief Scientific Advisers - The Science and Technology Committee. London. Recuperado de: https://publications.parliament.uk/pa/ld201012/ ldselect/ldsctech/264/26405.htm.

Piotrowski, J. (2014). «Ciencia lucha por ocupar un lugar en borrador de ODS ». SciDev.Net, 25 de julio. Recuperado de: https://www.scidev.net/americalatina/odm/noticias/ciencia-lucha-por-ocupar-un-lugar-en-borradorde-ods.html.

Ramírez Arroyo, R., H. Cahuas Miller y D. Pacheco Roldán (2012). Estadistica Forestal del Perú 2000-2010. Lima. Recuperado de: https://www.serfor. gob.pe/wp-content/uploads/2016/03/estadistica-2000-2010.pdf.

SERFOR (2015). Interpretación de la dinámica de la deforestación en el Perú y lecciones aprendidas para reducirla. Recuperado de: https://www. serfor.gob.pe/wp-content/uploads/2016/03/Interpretacion-de-ladinamica-de-la-deforestacion-en-el-Peru-y-lecciones-aprendidas-parareducirla-1.pdf.

SERNANP (2018). Listado de Áreas Naturales Protegidas de Administración Nacional con Categoría Definitiva. Recuperado de: http://www.sernanp. gob.pe/documents/10181/165150/LISTADO+ANP+29.04.2019. pdf/4aa8000b-7104-4eb5-bf67-e09d1f6aee9e.

SFERTF (s.a.). The South Florida Ecosystem Restoration Task Force. Recuperado de: https://www.evergladesrestoration.gov/tf/.

SFWMD (s.a.). «Our Work: the South Florida Water Management District». Recuperado de: https://www.sfwmd.gov/our-work.

Stevens Institute of Technology (s.a.). College of Arts and Letters, Science Communication Bachelor's Degree. Recuperado de: https://www. stevens.edu/college-arts-and-letters/undergraduate-programs/sciencecommunication.

The 106Th US Congress. (2000). «Public Law 106 - 541 - Water Resources Development Act of 2000». Recuperado de: https://www.govinfo.gov/ content/pkg/PLAW-106publ541/pdf/PLAW-106publ541.pdf.

The Everglades Foundation (s.a.). «The Everglades: Threats to the Ecosystem». Recuperado de: https://evergladesfoundation.org/the-everglades/ threats-to-the-ecosystem/. 
Universidad Autónoma de Madrid (2017). Curso de Experto en Comunicación Pública y Divulgación de la Ciencia. Recuperado de: https://www.uam. es/UAM/Curso_Experto_2017/1446726550625.htm?language=en\&pi d=1242652873305\&title=Curso de Experto en Comunicación Pública y Divulgación de la Ciencia.

University of Maryland (s.a.). Center for Environmental Science/Resources. Recuperado de: https://www.umces.edu/ian/science-communication/ resources.

USAID - NASA. (2017). RFA-530-17-000001. SERVIR Amazonía.

Von Winterfeldt, D. (2013). «Bridging the gap between science and decisionmaking». Proceedings of the National Academy of Sciences 110 (Supplement3), 14055-14061. Recuperado de: https://www.pnas.org/ content/110/Supplement_3/14055. 